\title{
Gold Contact Materials for Connectors
}

\author{
FACTORS AFFECTING THEIR SELECTION AND DESIGN
}

\section{George Tembe}

Johnson Matthey \& Co. Limited, London

The enormous demand for printed circuit edge connectors for use in telecommunications and in computers has led to the development of materials that offer several interesting routes by which production rates can be increased and economies effected. This article, based upon a paper presented to "Inter/Nepcon 71", briefly describes these materials and suggests how they can best be utilised.

With many possible combinations of contact material and type of construction from which to choose, it is important that connector designers are fully aware of what is available to them so that they can employ the most economically convenient form for each specific design. A review of available materials and their associated processing techniques may also lead to new and less costly designs of connectors.

\section{Electroplating}

Gold plating is of course widely used in the production of connectors. Generally, the parts that are to be plated are interconnected by wiring and are attached to a supporting jig or busbar. In other cases, if the parts are robust and are not inclined to tangle, the barrel-plating technique may be used. With this latter method, however, it is necessary to immerse the parts totally and therefore to deposit gold all over their surfaces. But with the former technique, it may be possible to arrange the wiring and jigging so that the parts need not be totally immersed, so saving on the consumption of gold.

Wiring and jigging (not to mention un-wiring and un-jigging) are laborious and costly operations. By obtaining parts already interconnected, plating costs can be substantially reduced. An increasingly popular technique, therefore, is to design the tools and method of fabrication of connectors so that the parts come off the press linked together in a continuous band, all part of the original strip material, as shown in Fig. 1. Cropped lengths off such a band
Fig. 1 To avoid the wiring and jigging of individual parts for gold plating one technique is to design the press tools to produce connectors in the form of a continuous band. Cropped lengths can then be gold plated and afterwards separated for assembly into mouldings. Two types of linked connector parts made hy Carr Fastener are sliown lere after plating and before separation and assenubly

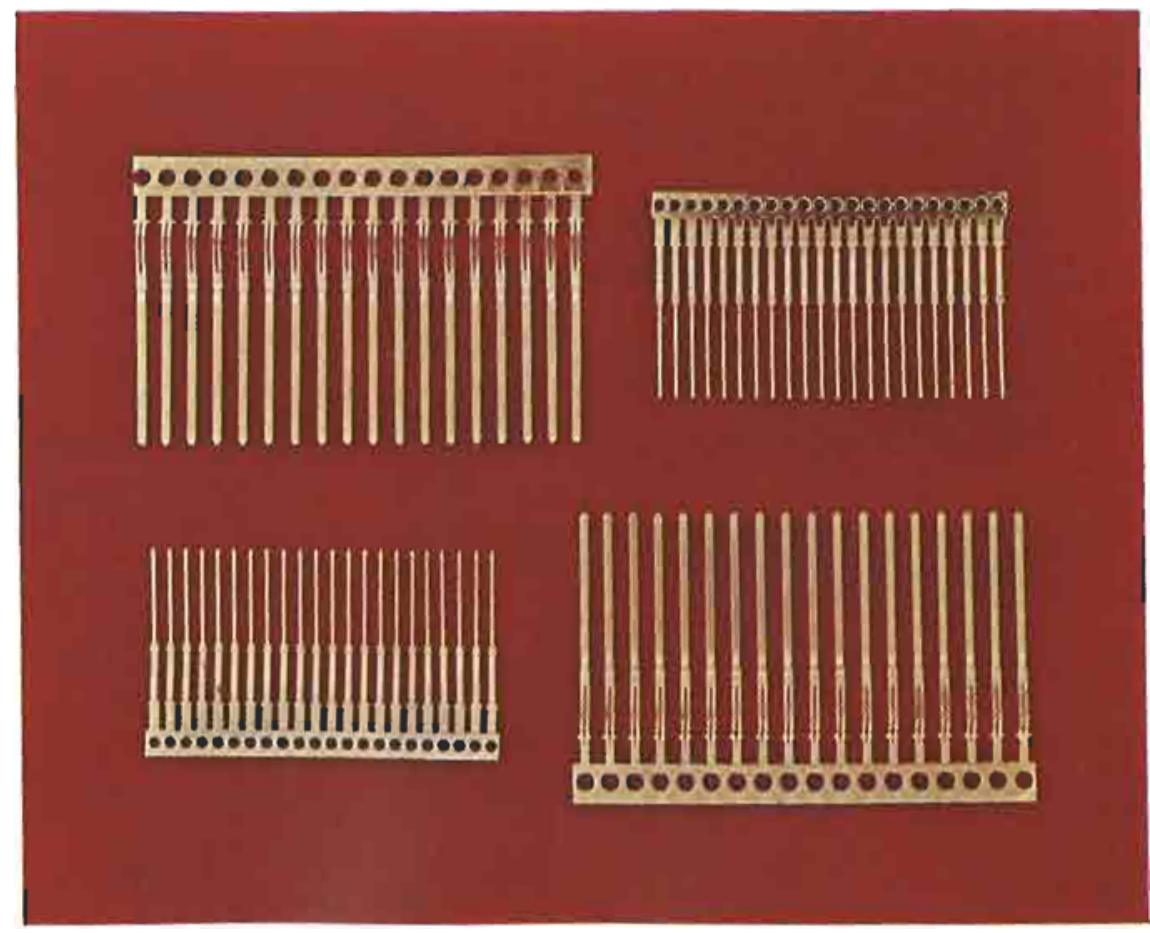


Fig. 2 One means of restricting gold to a required contact area only has been made possible by the production of long coiled strips of phosphor bronze or nickel silver with a stripe of electroplated gold rumning along its entire lengtl.. If necessary, more than one stripe can be proluced on one or both faces, as shown in the plotograpli of strips manufactured by N. T. Frost

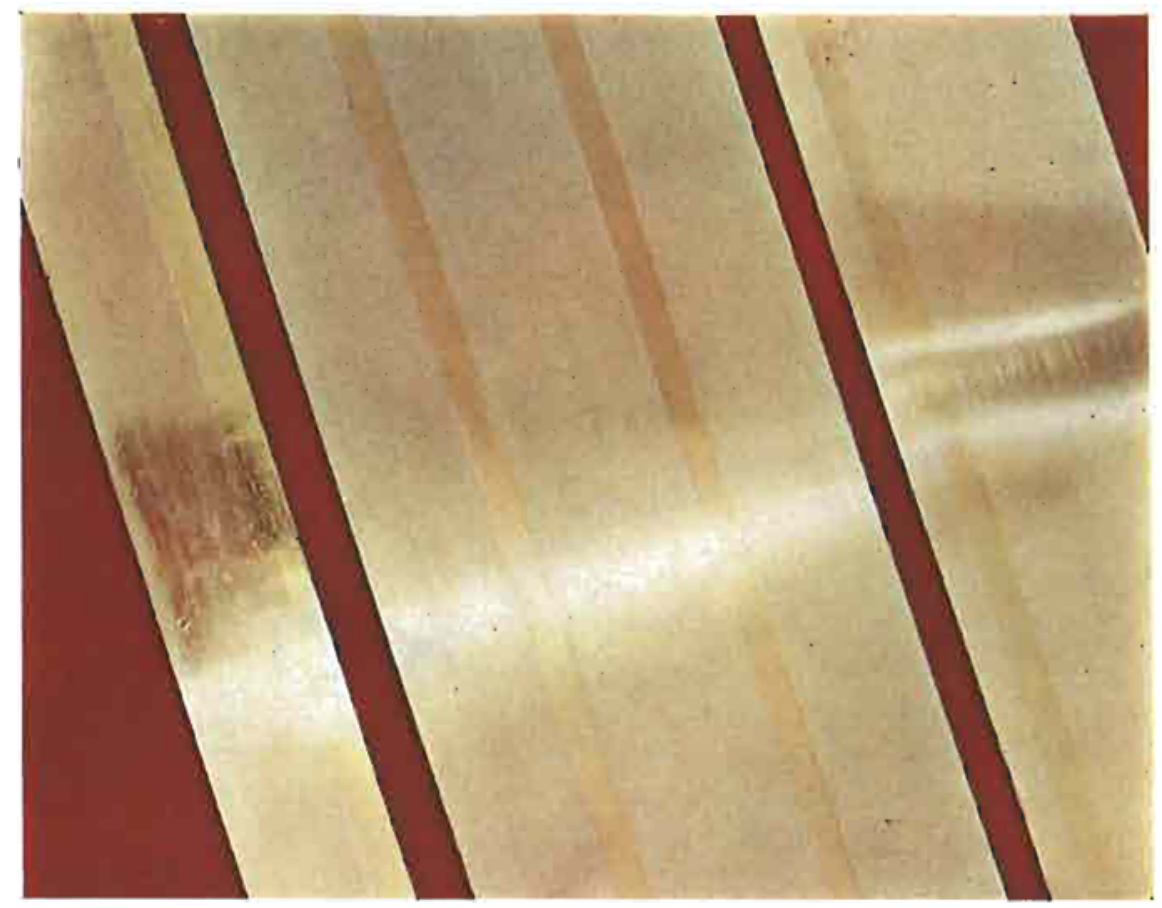

of parts can then be conveniently plated in a vat and the individual parts can be separated afterwards. Linked parts make for automated handling not only from the plating point of view but also in ease of assembling connector parts in mouldings.

To restrict the area over which gold is to be electrodeposited on a fabricated part requires some ingenuity and patience. Masking (and unmasking) areas of a part that do not require gold is timeconsuming no matter how it is contrived. The labour costs involved can rarely be less than the value of the gold saved, and are generally more. What is required ideally is a quick and simple method by which gold can be restricted to a required area. To gain the benefit from the gold that is saved speed is the essential factor and sufficient speed cannot be achieved on finished, fabricated parts.

A long coiled strip in, say, phosphor bronze or nickel silver can be produced with a stripe of electroplated gold running along its entire length. The stripe can be so positioned, and be of an adequate width, to ensure that only the contact area of the eventual fabricated part is covered with gold. If required, more than one stripe can be provided on one or both strip faces. Typical designs of stripe-plated strip are shown in Fig. 2.

In utilising strip material that has a continuous gold stripe it is almost inevitable that some of that restricted amount of gold will still be scrapped. To reduce this gold loss one would like to have regular spaces in the gold to coincide with the scrap portions in the blanked strip. This can be achieved by a further sophistication of the masking and continuous plating technique, so that strip material can be produced with regularly spaced, accurately located, gold plated areas - "dots and dashes", as illustrated in Fig. 3.

The processing of strip to produce lines of gold plated dots and dashes is essentially the same as for continuous stripe plating in that the areas not re-

Fig. 3 An alternative method of employing continuously plated strip is achieved by a further development, the production of regularly spaced and accurately located gold plated areas

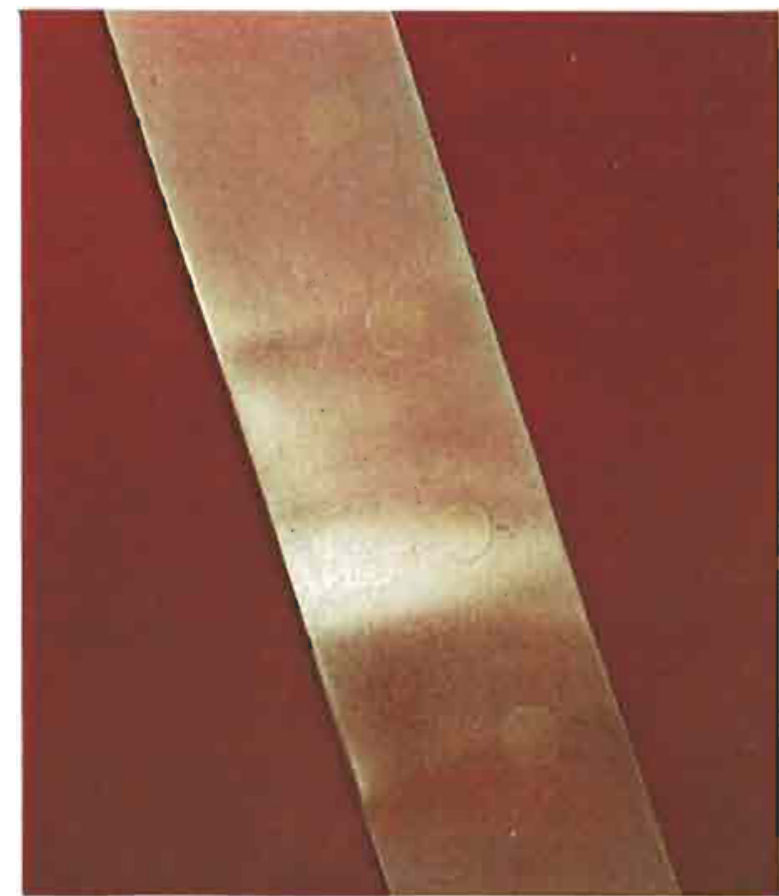


quired to be plated are masked from the electrolyte and the strip passes through all the stages in one run.

In both categories of continuous plated strip a variety of types of gold deposits are available. These range from fine golds of 60 to 100 Vickers hardness to "hard" golds of 150 to 200 Vickers and, even beyond, to alloyed golds of around 300 . The increasing hardness of these gold deposits tends to be associated with increasing inherent stresses in them and, therefore, with decreasing ductility, so that a choice must depend to some extent upon the formed shape the gold may have to take in fabrication. In some cases it is possible to have a primary deposit of nickel under the gold.

At first sight it may appear that, when faced with the various gold plating routes available for connector parts, the last described - continuous dots and dashes - is the ultimate cost-saver. But this is not necessarily so. The apparent saving in gold is not the only criterion. For example, it is more expensive to set up to produce dots and dashes than to produce continuous stripe, just as higher capital costs are incurred in setting up for continuous strip plating than for plating parts in a vat. Therefore, only if the value of the gold saved more than compensates for the higher manufacturing costs is a more sophisticated process viable. Generally this is strongly related to the throughput for a production run, but the design of the connector part and the positioning, thickness and type of gold deposit also can have a significant bearing upon the eventual decision. But it must be remembered that gold plating may not be the best answer in any case.

\section{Inlaid Contact Bi-metal Strip}

Gold inlaid contact bi-metal strips consist of such base metal materials as phosphor bronze and nickel silver, inlaid with a continuous stripe of a gold alloy or fine gold. The minimum width of an inlaid stripe is about $3 \mathrm{~mm}$ and the minimum thickness can be about $2 \mu \mathrm{m}$.

A manufacturing process for bi-metals starts with a block of base metal into which is bonded one or more inlays of fine gold or of a selected gold alloy with an interlayer of nickel if required. The composite block is then progressively rolled down to the final required strip thickness and is then slit to the required width. The final requirements for overall width and thickness, and for the gold inlay width and thickness, determine the various dimensions and ratios of the original composite block. The inlaid stripe so produced is firmly bonded to the base metal along its base and its edges.

A bi-metal combination that will suit a specific need can usually be obtained, and the choice allows a connector designer to establish and specify the most economic gold alloy in terms of environmental and mechanical performance. As a broad rule 5 per cent nickel-gold can be regarded as equivalent to a "hard" gold electrodeposit. Gold alloys with 70 to 75 per cent gold content are the least costly that will withstand the 20-day Industrial Atmosphere Test for the British Post Office. Alloys of progressively lower gold contents are increasingly more prone to corrosion and are therefore of decreasing reliability, yet are sometimes suitable to meet the requirements of low cost commercial connectors that do not have to pass very searching tests.

The range of available gold alloys also allows a designer to make a choice with, perhaps, other considerations in mind. For example, the ductility or hardness of the alloy may be a factor that will influence the choice and it is possible therefore to opt for a certain gold content and certain alloying constituents that together will provide the right physical properties from the point of view of fabrication of the connector parts and/or their subsequent performance characteristics. Fig. 4 shows two sets of parts manufactured from inlaid contact bi-metal strips. In both cases the strip is of $0.5 \mathrm{~mm}$ thick phosphor bronze inlaid with $5 \mu \mathrm{m}$ thick 25 per cent silver-gold. Assemblies incorporating such components have received Post Office approval.

One or more inlaid stripes can be produced in a gold bi-metal and it is possible to inlay both strip faces if required. Though the need is mostly for inlaid gold, it is, of course, possible to have the gold all over a strip face.

\section{Contact Materials for Welding}

In this category we are concerned with connector parts that are manufactured by welding to the spring arm a discrete piece of prefabricated contact material, Contact materials for this type of work are used in three main forms: wires, spheres and tapes.

Wires can be used in one of two ways. One way is to weld one end of a length of wire vertically to the backing, crop the wire close to the weld so as to leave a small lump of contact material in position and, finally, coin this lump to produce the finished, workhardened contact blended with the curvature of the connector arm. The other method uses short cropped pieces of wire that are transferred on to the connector arm and welded horizontally in position, followed by a mild coining operation to obtain the correct profile and height. For both cases, there are special machines available on the market that carry out the operations automatically or semi-automatically, either on dial- or magazine-fed parts or as an in-line process on webbed parts or continuous strips.

The second form, mentioned earlier, in which contact materials are used to make welded assemblies, 
Fig. 4 The use of gollinlaid contact bi-metal strip elvables the designer to select the most economic gold alloy iil terms of the required performance as well as tle method of fabrication. These pling and socket parts manufactured by Plessey Telecommunications are stamped from inlaid bi-metal of 25 per cent silver-gold in phosphor brouze and are used on panel nonmted circuits and rack slielves in the Pentex electronic telephone exchanges

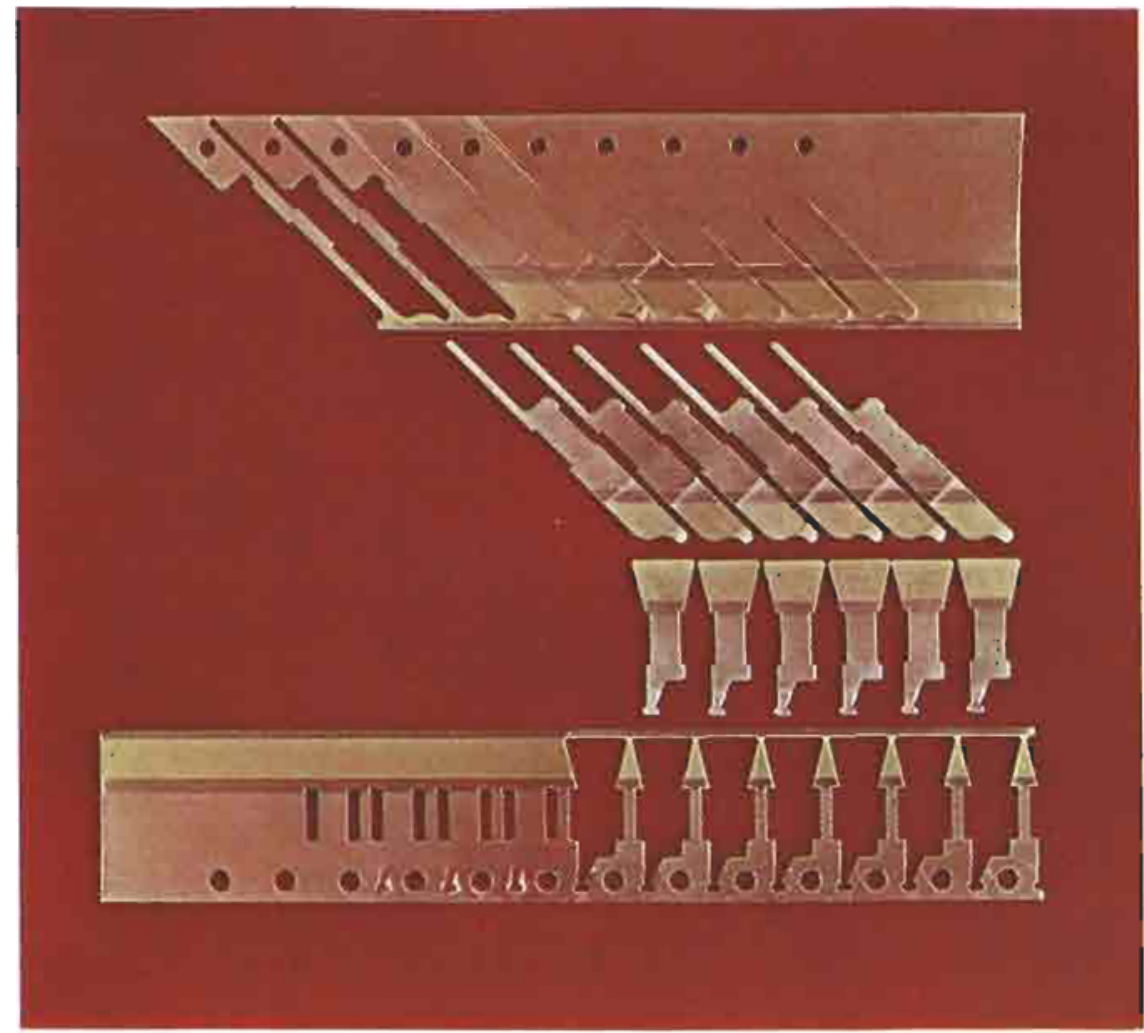

is that of spheres. For this purpose, small spheres typically $0.3 \mathrm{~mm}$ diameter - are available in fine gold and a range of gold alloys. High speed machines feed, weld and coin spheres generally in an in-line process where the backing material, usually in the form of rectangular wire, comes off a large coil. The coining station often incorporates the forming and cropping tools for the connector parts.

The third form in which a contact material for welded connector assemblies can be used is derived from a technique that has been well known for many years in the U.S.A., particularly, and in Japan, in the manufacture of numerous types of relays and small switches and for which there is now a fast growing interest in Europe. The material is used in the form of a narrow bi-metallic tape in which the top layer is of a selected contact material, such as a gold alloy and the bulk is a base metal, usually nickel or a cupro-nickel alloy.

A typical bi-metallic tape could be about $0.7 \mathrm{~mm}$ wide at the base with an overall height of about 0.4 $\mathrm{mm}$ which would include a contact layer of, say, 0.1 $\mathrm{mm}$ thickness or more or less if required. The top surface could be flat but is more likely to be radiused to suit the curvature of the eventual connector part. Along the base of the tape there would usually be a hemispherical bead of about $75 \mu \mathrm{m}$ radius, centrally located and running along the whole tape length. This bead provides the welding projection for each little contact that will be cropped off the tape.
In the assembly process, cropped pieces from a long continuous reel of tape are transferred on to the backing spring and welded in position. The backing can be presented as discrete connector parts or as a continuous strip for an in-line process. A final coining or sizing operation may then be carried out, although the shape of the tape has been manufactured already to suit the connector part. There are certain machines available for manufacturing welded tape assemblies, although a custom-built model is usually needed to suit a specific job.

Of the three basic forms of welded contact assemblies, the last one described here is a fairly recent development for connectors, although well established in other fields. It requires, however, a fairly large capital outlay to set up and the consumption of a single tape design must also be high to gain a real material cost saving because production costs for tapes are relatively high when compared with conventional wires, and even with spheres. The same considerations also apply to the other welding methods. These are all essentially processes that are economic only for long-running, repetitive production of individual components.

\section{Profiled Strips}

The last type of contact material that will be briefly described is the profiled strip for the manufacture of connectors. There are many designs of connectors that incorporate in their cross-section two or more 


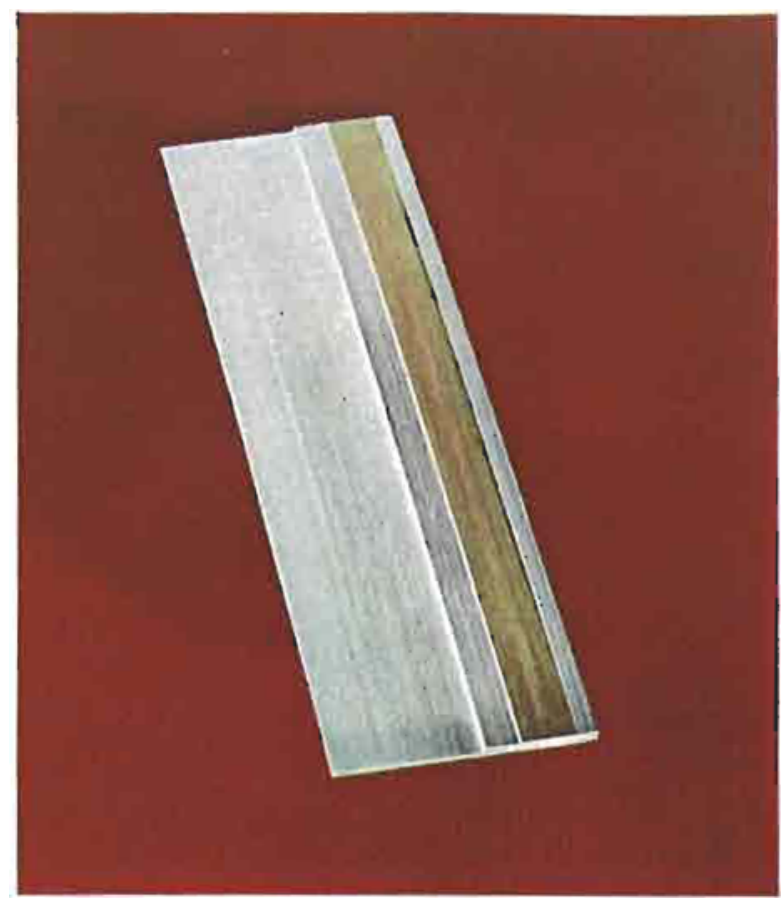

thicknesses of material that make up the backing for the contact. One object of such designs is to provide a flexible spring arm that is supported by, or merely attached to, a stouter terminal end. By using profiled strips such parts can be manufactured in one piece, eliminating joints or the need to deform the metal to produce the thinner section or sections.

All the various combinations of inlaid contact bimetals described earlier can be obtained in the form of profiled strips. In producing profiled sections one starts with normal bi-metal strip and merely removes metal that is excess to requirement. By this means an abundant variety of inlaid bi-metal profiled strips can be produced, one of which is illustrated in Fig. 5 .
Fig. 5 Many types of connectors incorporate two or more thicknesses of metal in their cross scction. The development of profiled bi-metal strip makes it possible to manufaeture such parts in a single press operation. This is only one example of a range of profiled strips being produced by Jolinson Matthey Metals and comprises an inlay of $10 \mathrm{per}$ cent copper-20 per cent silver-gold in nickel silver

Profiled strips are already being used to produce connectors, but the full scope of the process and its potential for creators of new connector designs has yet to be exploited.

\section{Conclusion}

The object of this paper has been to present a brief review of the forms in which gold contact materials are available and are being used for the production of connectors, and thereby to stimulate designers and manufacturers to fresh ideas. The varieties of gold contact forms are numerous and it may be possible to detect from this survey the possibility of an immediate cost reduction on an established connector, especially where an economic substitution can be made with little or no inconvenience or modification to the established manufacturing process and tooling.

However, the time to consider all these possible permutations is long before production has been commissioned. That is the time to take advice on what specifically is available, and to juggle with the alternatives. Only in this way can one arrive at the best economic choice that will match the design, the fabrication and the performance of a connector.

\section{The Durability of Plated Electrical Contacts}

\section{DIFFUSION OF SUBSTRATE COPPER INTO GOLD DEPOSITS}

The use of gold-plated copper in electrical contacts is well established but there have recently been trends towards thinner plating and subsequent operation at higher temperatures than in the past. There has been a consequent tendency for copper to diffuse to the surface of the gold, where it becomes oxidised and forms an insulating film which interferes with good contact. A method of assessing the life of gold-plated contacts affected by this mechanism would therefore be a useful guide to service behaviour.

M. R. Pinnel and J. E. Bennett of Bell Laboratories, Columbus, Ohio have now reported studies leading to such a method for temperatures below $250^{\circ} \mathrm{C}$ at the Holm Seminar on Electric Contact Phenomena held in Chicago during October 1971. Diffusion rates were measured for $2.5,25$ and $100 \mu$ gold plating on poly- crystalline copper during annealings at temperatures between 50 and $750^{\circ} \mathrm{C}$. Chemical interdiffusion coefficients were found to deviate from predicted values below $250^{\circ} \mathrm{C}$. They were significantly greater below $150^{\circ} \mathrm{C}$. There was no correlation between plating thickness and diffusion rate.

These diffusion coefficients can be used to estimate the time at each temperature which it takes before degradation of the electroplated gold surface occurs by mass diffusion of the copper substrate. Studies with 1 and $2.5 \mu$ gold layers during degradation to 0.1 and 1 per cent surface copper concentrations show, for example, that at $65^{\circ} \mathrm{C}$ it takes as long as seven years for the surface copper concentration of a $2.5 \mu$ gold layer to reach 0.1 atomic per cent and twelve years to reach the 1 atomic per cent level. 